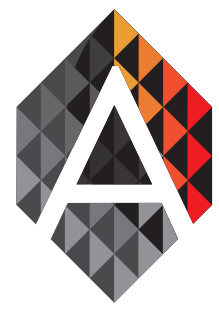

\title{
Data-Mining-based filtering to support Solar Forecasting Methodologies
}

\author{
Tiago Pinto ${ }^{\mathrm{a}}$, Luis Marques ${ }^{\mathrm{b}}$, Tiago M. Sousa ${ }^{\mathrm{b}}$, Isabel Praça ${ }^{\mathrm{b}}$, \\ Zita Vale ${ }^{\mathrm{b}}$ and Samuel L. Abreu ${ }^{\mathrm{c}}$ \\ aBISITE research group, University of Salamanca, Calle Espejo, s/n, 37007 Salamanca, Spain, tpinto@usal. \\ es \\ ${ }^{\mathrm{b}} \mathrm{GECAD}$ - Research Group on Intelligent Engineering and Computing for Advanced Innovation and \\ Development - Polytechnic of Porto (ISEP/IPP), R. Dr. António Bernardino de Almeida, 431, \\ P-4249-015 Porto, Portugal, \{luma, tmsbs, icp, zav\}@isep.ipp.pt \\ 'General - Alternative Energies Group - IFSC - Instituto Federal de Santa Catarina, abreu@ifsc.edu.br
}

\section{KEYWORD}

Artificial Neural

Network;

Clustering, Data

Mining; Machine

Learning; Solar

Forecasting;

Support Vector

Machine

\section{ABSTRACT}

This paper proposes an hybrid approach for short term solar intensity forecasting, which combines different forecasting methodologies with a clustering algorithm, which plays the role of data filter, in order to support the selection of the best data for training. A set of methodologies based on Artificial Neural Networks (ANN) and Support Vector Machines (SVM), used for short term solar irradiance forecast, is implemented and compared in order to facilitate the selection of the most appropriate methods and respective parameters according to the available information and needs. Data from the Brazilian city of Florianópolis, in the state of Santa Catarina, has been used to illustrate the methods applicability and conclusions. The dataset comprises the years of 1990 to 1999 and includes four solar irradiance components as well as other meteorological variables, such as temperature, wind speed and humidity. Conclusions about the irradiance components, parameters and the proposed clustering mechanism are presented. The results are studied and analysed considering both efficiency and effectiveness of the results. The experimental findings show that the hybrid model, combining a SVM approach with a clustering mechanism, to filter the data used for training, achieved promising results, outperforming the approaches without clustering.

\section{Introduction}

Governmental policies and incentive programs at a worldwide scale are promoting the increase of renewable energy generation, with the aim of reducing greenhouse gas emissions and decreasing the dependency on fossil fuels. In Europe, a set of legislation has been defined having the known «20-20-20» as targets (European Commission, 2017). The national targets will enable the $\mathrm{EU}$ as a whole to reach its $20 \%$ renewable energy target for 2020 - more than double the 2010 level of 9.8\%. These targets, which reflect Member States' different starting points and potential for increasing renewables production, range from $10 \%$ in Malta to $49 \%$ in Sweden (European Commission, 2017).

Tiago Pinto, Luis Marques, Tiago M. Sousa, Isabel Praça, Zita Vale and Samuel L. Abreu Data-Mining-based filtering to support

Solar Forecasting Methodologies
ADCAIJ: Advances in Distributed Computing and Articial Intelligence Journal Regular Issue, Vol. 6 N. 3 (2017), 85-102 elSSN: 2255-2863 - http://adcaij.usal.es (c) Ediciones Universidad de Salamanca - CC BY 
In order to meet such ambitious goals, renewable and clean energy sources, such as tidal, wind and solar have become of great importance. However the variable and intermittent nature of these resources poses a lot of challenges to several entities such as utility companies, power systems operators and market operators, especially when considering a significant market penetration rate as it is expected and encouraged to achieve (Sioshansi, 2013).

Solar energy is clearly the most abundant resource available to modern societies. Usually summer months, such as July and August in the northern hemisphere, have smaller variability. However, even during some sunshine months sudden changes might occur. The variability of the solar resource is mostly due to cloud cover variability and atmosphere conditions (Martin et al., 2010).

Due to its particular characteristics, several approaches are usually used to forecast solar intensity, namely physical models (Badescu, 2008), time series analysis (Paolik et al., 2009), and other forecasting algorithms, such as reviewed in (Inman et al., 2013). Artificial Neural Networks (ANN) (Keles, 2016) and Support Vector Machines (SVM) (Pinto et al., 2014), (Pinto et al., 2016) are some of the most widely used techniques, and have been used for time series forecasting in several domains. Current approaches lack, however, a contextualization of the problem, so that the forecasting process is able to understand which is the most important data that should be used in each case. This leads to problems regarding the forecasting accuracy (by using irrelevant or even misleading data in the training process), and also to the efficiency of the forecasting process, since using large amounts of unnecessary data hardens the training process and leads to larger execution times.

A relevant step towards overcoming the gap in the literature has been achieved in (Pinto et al., 2015), where a study is conducted, in order to identify similar hours of solar intensity, according to different perspectives. The automatic adaptation of the forecasting approaches and of the used variables according to the different types of hours, is however, still not addressed. It is in this scope that this paper provides its contribution, by conducting a study with the aim of understanding and improving the solar irradiance forecasting, given its particular variability. For this, several forecasting methods, based on ANN and SVM are used and conclusions about the solar irradiance components and algorithms' parameters are taken. An hybrid approach is also proposed, which combines ANN and SVM with a clustering algorithm, which is used to filter the data that is most appropriate to be used in the training process of the forecasting methodologies. The proposed hybrid approach proves to be an interesting area of research, capable of improving ANN or SVM results both in terms of forecasting effectiveness and execution time efficiency by selecting only the most correlated data (most similar hours and days) as training data for the forecasting methods. The use of the specific historical data that most potentiates the optimization of the forecasting methods proves to have an equal or even higher importance than the optimization of the methodologies' parameters themselves.

Section 2 presents an overview of the related work, including a discussion on solar irradiance components and the importance of its forecast, and highlighting the current state of the art related to solar intensity forecasting. Section 3 presents the ANN and SVM techniques that are used in this work, and details the clustering model that has been used to implement the proposed hybrid approach for solar forecast. In section 4 experimental findings on the proposed methods and the obtained conclusions are presented and discussed. Real data from solar irradiance of Florianópolis, in Santa Catarina, Brazil areused in the experiences. Finally, section 5 presents the most relevant conclusions and contributions of this work.

\section{Solar Forecasting}

This section reviews the most relevant work related to solar intensity forecasting, including a discussion on the importance of this type of prediction, a description of the most important parameters, and the review of current state of the art approaches to solve this problem, with special emphasis on hybrid forecasting approaches. Finally a discussion is provided, highlighting the main limitations of current approaches, and the need for novel contributions that address such limitations.

Tiago Pinto, Luis Marques, Tiago M. Sousa, Isabel Praça, Zita Vale and Samuel L. Abreu Data-Mining-based filtering to support

Solar Forecasting Methodologies
ADCAIJ: Advances in Distributed Computing and Articial Intelligence Journal Regular Issue, Vol. 6 N. 3 (2017), 85-102 eISSN: 2255-2863 - http://adcaij.usal.es (c) Ediciones Universidad de Salamanca - CC BY 


\subsection{The importance of solar intensity forecasting}

Despite its importance for the existence of life on earth, and human beings health, the sun is nowadays a source of clean energy and can contribute to reduce the difficulty in fulfilling the energy demand. Photovoltaic (PV) and solar thermal are the main sources of electricity generation from solar irradiance. In the case of solar thermal energy plants with storage energy system, its management and operation need reliable predictions of solar irradiance with the same temporal resolution as the temporal capacity of the back-up system (Martin et al., 2010). The development in the power semiconductor technology has allowed higher efficiencies in the conversion of solar energy into electrical energy trough photovoltaic cells (Gupta et al., 2010) and PV systems have reached the end-user. The spread of PV technology took place and nowadays is being used in several buildings to generate electricity.

The increase on the use of renewable energy sources (RES) affects the behaviour of a considerable number of entities from the electricity sector and imposes economical and technical challenges. Forecasting renewable resources is important from the producers, retailers, aggregators, system operators and market operators' point of view.

From the utility point of view, application of renewable sources can potentially reduce the demand for distribution and transmission facilities. Clearly, distributed generation located close to loads can reduce power flows in transmission and distribution circuits with two important effects: loss-reduction and the ability to potentially substitute for network assets. Furthermore, the presence of generation close to demand could increase service quality seen by end customers (Schwaegerl and Tao, 2013).

From the power system operators' point of view, short-term forecasting is relevant for dispatching and regulatory purposes, to optimize the decision making by allowing corrections to unit commitment.

Concerning market operators, the prevision of the production is important for planning the transactions in the electricity market in order to assure the balancing between supply and demand. From the economical point of view it is also important for electricity players to use this knowledge as competitive advantage in day-ahead electricity trading.

The balancing market is a complementary market to the day-ahead market, which allows agents to adjust their needs and renegotiate previously agreed energy by adjusting the quantities traded in the daily market. This enables players to overcome fluctuations of the production forecasts, which is particularly important for producers based on RES, such as wind and solar power, due to their variable and intermittent nature. Solar, wind and load forecasting have become integral parts of the smart grid and microgrid concepts.

\subsection{Solar irradiance components}

The solar irradiance fluctuates around an average value of approximately 1360 Wm-2 (Kopp and Lean, 2011). The incident extra-terrestrial beam radiation is divided in two distinct components: the Direct Normal Irradiance (DNI) and the Diffuse Horizontal Irradiance (DHI). The geometric sum of both results is the Global Horizontal Irradiance (GHI) that can be written as (1)

$$
G H I=D H I+D N I * \cos \theta
$$

where $\theta$ is the solar zenith angle. When the sun is directly overhead $\theta=0$ and means we are calculating the extra-terrestrial radiation that reaches a surface perpendicular to the sun.

The extra-terrestrial irradiation is measured above the Earth's atmosphere, so it is not influenced by clouds in the atmosphere and can easily be previewed throughout the year (Inman et al., 2013).

\subsection{Solar forecast}

Usually to predict renewable sources of energy two approaches may be used: an approach based on physical models (Badescu, 2008), using mathematical equations to describe physics and dynamics of the atmosphere that influences solar radiation, and an approach based on time series analysis by means of statistical models (Paolik

Tiago Pinto, Luis Marques, Tiago M. Sousa, Isabel Praça, Zita Vale and Samuel L. Abreu Data-Mining-based filtering to support

Solar Forecasting Methodologies
ADCAIJ: Advances in Distributed Computing and Articial Intelligence Journal Regular Issue, Vol. 6 N. 3 (2017), 85-102 elSSN: 2255-2863 - http://adcaij.usal.es (c) Ediciones Universidad de Salamanca - cc BY 
et al., 2009). Physical models work well for medium- and long-term solar forecasting, while statistical models have lower complexity and can perform well for short-term solar intensity forecasting.

Several techniques have been applied to solar irradiation or solar power forecast such as regression techniques, Auto Regressive Moving Averages (ARMA), Auto Regressive Integrated Moving Averages (ARIMA), Artificial Neural Networks (ANN), Genetic Algorithms (GA) and Support Vector Machines (SVM). References (Inman et al., 2013), (Pelland, 2013), (Diagne et al., 2013), (Mohanty et al., 2017) provide good overviews on the current state of the art in solar irradiance forecasting, while (Voyant, 2017) provides a more specific review on the application of machine learning methods for solar radiation forecasting. The work proposed in (Barzin, 2016) suggests that the use of gradient boosted regression trees can be a valid solution for multi-site solar power forecasting. In (Persson, 2017) solar forecasting is used as basis for a price-based control mechanism for PV. In (Pedro and Coimbra, 2012) a comparison on several forecasting techniques to predict solar power at a photovoltaic power plant in California is presented. In this work, ANN has proved to be a promising technique on this field, showing improved results while combined with GAs. The same conclusions about the use of ANNs were achieved by (Ioakimidis, et al., 2013) and (Singh et al., 2013). ANNs have also been successfully applied to the forecasting of other renewable sources based production types, such as the wind power, in (Hao et al., 2014). The good results achieved by ANNs in the most varied fields (Huynh and Reggia, 2012), (Wilamowski and Yu, 2010), (Bian and Chen, 2014) and (Dianhui and Tapan, 2013), provide an encouraging indication of ANNs' capability of coping with the problem approached in this work.

In (Sharma et al., 2011) a machine learning approach is proposed to forecast solar generation. The use of a weighted SVM methodology to forecast solar power is proposed in (Xu et al., 2012), and (Zeng and Qiao, 2013) presents an alternative SVM method to predict solar power. SVM has proved to be a promising technique for solar forecasting research.

The work presented in (Alessandrini et al., 2015) proposes the application of an analog ensemble (AnEn) method to generate probabilistic solar power forecasts. The AnEn is based on an historical set of deterministic numerical weather prediction model forecasts and observations of the solar power.

Despite the relevant developments that have been achieved so far, the amount of data that is nowadays available to be used by forecasting algorithms, together with the variability of the associated information, and the necessity for correlating different types of data from different sources, makes the most typically used approaches unable to cope with the current needs.

In order to enable a breakthrough in the field, hybrid methodologies that combine the best features of different approaches, are arising. A novel hybrid model for hourly solar radiation forecasting, based on the combination Mycielski-Markov is proposed in (Hocaoglu, Serttas, 2017). A multi-step forecasting method based on the combination of weather research and forecasting ensembles, a novel fuzzy system, and a cuckoo search algorithm is proposed in (Zhao et al., 2016), for operational wind forecast. Also applied to wind forecasting, (Liu et al., 2015) proposes a new hybrid approach based on the Secondary Decomposition Algorithm (SDA) and the Elman neural networks. A hybrid load forecasting model with parameter optimization is proposed for short-term load forecasting in (Liu et al., 2014), being composed of Empirical Mode Decomposition (EMD), Extended Kalman Filter (EKF), Extreme Learning Machine with Kernel (KELM) and Particle Swarm Optimization (PSO).

\subsection{Conclusions}

Although the advances in this field account for many relevant contributions, the requirements in terms of forecasting quality and execution time makes current algorithms insufficient to assure the level of responsibility that is attributed to forecasting methods. The existing approaches make use of huge amounts of data, which on one hand, limits the ability to extract relevant knowledge from the available information, due to the amount of unnecessary and misleading data, and on the other hand makes current models too heavy to be able to provide reliable forecasts in a fast execution time.

For these reason, there is an increasing need for data filtering methods that, through their combination with forecasting algorithms are able to select the data that most matters for each application context. This way, correlations in data can be found in an early stage of the process and irrelevant data can be discarded from the

Tiago Pinto, Luis Marques, Tiago M. Sousa, Isabel Praça, Zita Vale and Samuel L. Abreu Data-Mining-based filtering to support

Solar Forecasting Methodologies
ADCAIJ: Advances in Distributed Computing and Articial Intelligence Journal Regular Issue, Vol. 6 N. 3 (2017), 85-102 eISSN: 2255-2863 - http://adcaij.usal.es (c) Ediciones Universidad de Salamanca - CC BY 
start, thereby contributing to the increase of forecast accuracy, through the use of the most relevant data in each context, and also boosting the decrease of execution times by reducing the amount of data for training.

The hybrid approach presented in this paper contributes to overcome the current identified limitations by proposing the combination of forecasting algorithms, namely ANN and SVM, with a clustering algorithm, which has the role of filtering the input data by finding associations in the full amount of data, according to each context. This data filtering allows identifying and selecting only the most appropriate data to be used for training in the forecasting algorithms.

\section{Proposed Hybrid Forecasting Methodology}

This section presents the solar forecasting and the artificial intelligence techniques that we have used in this work. Firstly, the forecasting algorithms considered in this work are presented, namely the ANN and SVM. The clustering approach that is proposed as data-filtering method do select the input data for the forecast process is also presented, and complemented by an overview of the forecasting accuracy measurement methods that are used in this study.

\subsection{Artificial Neural Networks}

Artificial Neural Networks (ANN) are inspired on the human brain and their amount of neurons with high interconnectivity. ANNs are constituted by several nodes or neurons, organized in different levels, and interconnected by numeric weights. They resemble to the human brain in two fundamental points: the knowledge being acquired from the surrounding environment, through a learning process; and the network's nodes being interconnected by weights (synaptic weights), used to store the knowledge. Each neuron executes a simple operation, the weighted sum of its input connections, which originates the exit signal that is sent to the other neurons. The network learns by adjusting the connection weights, in order to produce the desired output - the output layer values (Schaefer et al., 2007).

Based on a large number of correct examples ANN are able to change their connection weights until they generate outputs that are coincident with the correct values. This way, ANN are able to extract basic rules from data (Wilamowski and Yu, 2010).

\subsection{Support Vector Machines}

The first algorithm for pattern recognition has been proposed in 1936 by R. A. Fisher (1936). The SVM algorithm is implemented by a generalization of the nonlinear algorithm Generalized Portrait that has been created by Vapnik and Lerner (1963). This was the first running kernel of SVM, only for classification and linear problems.

The SVM concept can be tracked to when statistical learning theory was developed further with Vapnik, in 1979. However, the SVM approach in the current form was first introduced with a paper at the COLT conference, in 1992 (Boser et al., 1992). The information to use in an SVM must follow the format suggested in (2):

$$
\left(y_{1}, x_{1}\right), \ldots,\left(y_{i}, x_{i}\right), x \in R^{n}, y \in R
$$

Where each example $x i$ is a space vector example; $y i$ has a corresponding value; $\mathrm{n}$ is the size of training data. For classification: $y i$ assumes finite values; in binary classifications: $y i \in\{+1,-1\}$; in digit recognition: $y i \in\{1,2,3,4,5,6,7,8,9,0\}$; and for regression purposes, $y i$ is a real number $(y i \in \mathrm{R})$.

The implementation of SVM requires considering some important aspects, namely:

- Feature Space is the method that can be used to construct a mapping into a high dimensional feature space by the use of reproducing kernels. The idea of the kernel function is to enable operations to be performed in the input space rather than the potentially high dimensional feature space. Hence the inner product does not need to be evaluated in the feature space. This provides a way of addressing the curse

Tiago Pinto, Luis Marques, Tiago M. Sousa, Isabel Praça, Zita Vale and Samuel L. Abreu Data-Mining-based filtering to support

Solar Forecasting Methodologies
ADCAIJ: Advances in Distributed Computing and Articial Intelligence Journal Regular Issue, Vol. 6 N. 3 (2017), 85-102 elSSN: 2255-2863 - http://adcaij.usal.es (c) Ediciones Universidad de Salamanca - cc BY 
of dimensionality. However, the computation is still highly dependent on the number of training patterns, and a good data distribution for a high dimensional problem generally requires large training sets.

- Loss Functions. In statistics, the decision theory and machine learning, the loss function is a function that maps an event to a real number, representing some «costs» associated with the difference between the estimated and the actual data for an occasion. The purpose of this function is to modulate the input data, when applied to a training set, and then forecasting the values (or sorting). The loss function uses the forecast values and compares how much they deviate from the actual values, quantifying the deviation (Nikulin, 2001).

- Kernel Functions. The kernel functions, in general, are a set of algorithms for pattern examination. The main task is to find patterns and study the type of associations, in a particular pattern (e.g, groups, classifications, major components, correlations, classifications) for general types of data (such as sequences, text documents, sets of points vectors, images, etc). The kernel function approach the problem by mapping the data to a dimensional space, where each coordinate corresponds to a characteristic of each input value transforming the data into a set of points in Euclidean space. Some examples of kernels are (Smola and Schölkopf, 2004): Polynomial, Gaussian Radial Basis Function, Exponential Radial Basis Function, Multi-Layer Perceptron, Splines, B splines. The most applicable kernels for time series forecasting, as is the problem of solar forecasting, are the Radial Basis Function (RBF) and the exponential Radial Basis Function (eRBF). These two kernels are specifically directed to regression in time series data. The SVM approach takes as parameters:

- trainingLimit - limit number of training data;

- kernel - kernel that is used in the regression process;

- $\varepsilon$-insensitive - error that is permitted, i.e. the lower the value, the higher the exigency of the regression process;

- limit - limit of the kernel function;

- $\sigma$ - angle of the kernel function;

- offset - offset of the kernel function.

A suitable combination of these parameters is essential to achieve quality results. The most suitable combination is highly dependable on the characteristics and particularities of each distinct problem; therefore, an exhaustive sensitivity analysis must be performed for each application, in order to achieve conclusions on the best combinations of parameters that should be used.

\subsection{Data clustering algorithms}

When trying to discover knowledge from data bases, one of the first tasks is to identify groups of similar objects to carry out cluster analysis for obtaining data partitions.

Clustering is a data mining process that enables dividing a set of data into separate groups, according to the similarity between the observations data (Han and Kamber, 2006). Hence, from a good clustering process result several clusters containing observations with high similarity between them, and high dissimilarity between data associated to different clusters (Jain et al., 1999).

$\mathrm{K}$-means is one of the most widely used clustering algorithms, mostly due to its effectiveness in different domains (Jain, 2010). K-Means contemplates a set of $n$ observations $\left(x_{p}, x_{2}, \ldots, x_{n}\right)$, in which each is a $d$-dimensional real vector. The clustering process partitions the $n$ observations into $k(\leq n)$ clusters $C=\left\{C_{1}, C_{2}, \ldots, C_{k}\right\}$ so that the Within-Cluster Sum of Squares (WCSS) is minimized, as in (3).

$$
\min \sum_{i=1}^{k} \sum_{x \in C_{i}}\left\|x-\mu_{i}\right\|^{2}
$$

where $\mu_{i}$ is the mean of points in $C_{i}$, i.e. the cluster centroid.

Tiago Pinto, Luis Marques, Tiago M. Sousa, Isabel Praça, Zita Vale and Samuel L. Abreu Data-Mining-based filtering to support

Solar Forecasting Methodologies

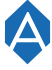

ADCAIJ: Advances in Distributed Computing and Articial Intelligence Journal Regular Issue, Vol. 6 N. 3 (2017), 85-102 elSSN: 2255-2863 - http://adcaij.usal.es (c) Ediciones Universidad de Salamanca - cc BY 
The vector that characterizes each observation $x_{p}, p \in\{1, \ldots, n\}$ is equal to the sum of the individual dimensions of $n$ vectors. Each $n$ vector comprises the information referring to a different variable. The clustering process undergoes an iterative process aiming at minimizing (3):

- Step 1 - each observation $x_{p}$ is assigned to cluster $C(t)$ whose mean value yields the minimum WCSS in iteration $t$, as presented in (4);

- Step 2 - the updated means are calculated for all clusters, considering the newly assigned observations, determining each cluster's new centroid $\mu_{i}$, as shown in (5).

$$
\begin{gathered}
C_{i}^{(t)}=\left\{x_{p}:\left\|x_{p}-\mu_{\mathrm{i}}^{(\mathrm{t})}\right\|^{2} \leq\left\|x_{p}-\mu_{\mathrm{j}}^{(\mathrm{t})}\right\|^{2} \forall j, 1 \leq j \leq k\right\} \\
\mu_{\mathrm{i}}^{(\mathrm{t}+1)}=\frac{1}{\left|C_{i}^{(t)}\right|} \sum_{x_{j} \in C_{i}^{(t)}} x_{j}
\end{gathered}
$$

The iterative process finishes when the convergence process is completed, i.e. when the observations assignment to distinct clusters stabilizes. Hence, the K-Means assigns observations to the nearest cluster by distance by minimizing the WCSS objective, in (3).

Determining the optimal value for $k$ is not an easy task, as it depends on the dispersion and scale of values and the envisaged clustering objectives. The optimal value of $k$ should guarantee the balance between concentrating all data in a unique cluster, and reaching maximum accuracy.

The Mean Index Adequacy (MIA) and Clustering Dispersion Indicator (CDI) (Chicco and Ilie, 2009) are methodologies that enable analysing the quality of observations distribution among distinct clusters. These approaches assess the dispersion of the observations among the clusters. MIA uses the Euclidean distance method to determine the value that reflects the quality of a cluster partition. CDI is determined by the association of the distance between elements of the cluster, and the inverse of the distance between the values that represent each cluster.

MIA depends on the average of the mean distances between each observation assigned to each cluster and the centroid of the corresponding cluster, as defined in (6).

$$
M I A=\sqrt{\frac{1}{K} \sum_{k=1}^{K} d^{2}\left(x^{(k)}, \mu^{(k)}\right)}
$$

CDI assesses: (i) the distance among the data points clustered in the same group, and (ii) the distance from each data point to all other clusters' centroids, as in (5), where $R$ is the mean point of all observations, and $n$ is the number of observations assigned to each cluster.

$$
C D I=\frac{\sqrt{\frac{1}{K} \sum_{k=1}^{K}\left[\frac{1}{2 . n^{(k)}} \sum_{n=1}^{n^{(k)}} d^{2}\left(x^{(m)}, \mu^{(k)}\right)\right]}}{\sqrt{\frac{1}{2 K} \sum_{k=1}^{K} d^{2}\left(x^{(k)}, R\right)}}
$$

The distance between two observations $x_{i}$ and $x_{j}$ is calculated as defined in (8).

$$
d(x i, x j)=\sqrt{\frac{1}{H} \times \sum_{h=1}^{H}(x i(h)-x j(h))^{2}}
$$

where $H$ is the size of the vector that contains all elements that represent each observation.

Tiago Pinto, Luis Marques, Tiago M. Sousa, Isabel Praça, Zita Vale and Samuel L. Abreu Data-Mining-based filtering to support Solar Forecasting Methodologies
ADCAIJ: Advances in Distributed Computing and Articial Intelligence Journal Regular Issue, Vol. 6 N. 3 (2017), 85-102 elSSN: 2255-2863 - http://adcaij.usal.es (c) Ediciones Universidad de Salamanca - cc BY 
The variance assessment provided by MIA and CDI for different numbers of $k$ enables analysing the gain (in variance reduction) of adding an extra cluster. When the variance from $k$ to $k+1$ is no longer significant and starts stabilizing, one has reached the optimal $k$.

\subsection{Accuracy of forecast}

The accuracy of the forecast may be evaluated by several error indices, such as the mean absolute error (MAE), the mean absolute percentage error (MAPE), the symmetric mean absolute percentage error (SMAPE) and the standard deviation (SD). MAE represents the average of the absolute errors (9).

$$
M A E=\frac{1}{N} \sum_{h=1}^{N}\left|Y_{h}-F_{h}\right|
$$

where $Y_{h}$ and $F_{h}$ are the actual and forecasted values for each $h$ period, while $N$ corresponds to the number of forecasted periods.

In MAPE the average of all the percentage errors is computed, producing a measure of relative overall fit (10).

$$
\text { MAPE }=\frac{100 \%}{N} \sum_{h=1}^{N}\left|\frac{Y_{h}-F_{h}}{Y_{h}}\right|
$$

A disadvantage of methods based on percentage errors is that they assume a meaningful zero. For example, they make no sense in measuring forecast error for temperatures on the Fahrenheit or Celsius scales (Hyndman and Koehler, 2006).

SMAPE (11) is an adaptation of MAPE, which is used to overcome the main restriction of the MAPE error, the impossibility of considering null values.

$$
S M A P E=\frac{1}{N} \sum_{h=1}^{N} \frac{\left|Y_{h}-F_{h}\right|}{\left(Y_{h}+F_{h}\right) / 2}
$$

\section{Experimental Findings}

This section shows the results of the tests that have been performed to assess the functioning of the methodologies and their parameters' tuning. All tests were performed for the same day and period, in order to conclude which is the most adequate methodology for the day and period under analysis.

This section is divided into five parts, namely: (i) results of the ANN based methodologies; (ii) results of the SVM based methodologies; (iii) results of the proposed hybrid approaches, including the clustering mechanism to filter the training data for the forecasting methodologies; (iv) execution times comparison; (v) results summary. This comparison allows reaching relevant conclusions about the best methodology to forecast solar intensity.

The used data are referent to Florianópolis, state of Santa Catarina, Brazil. These data correspond to the period from 1990 to 1999, including the values of Global, Direct, Diffuse and Extra-terrestrial Irradiance, in W/ $\mathrm{m} 2$; temperature in ${ }^{\circ} \mathrm{C}$; humidity in $\%$; and wind speed in $\mathrm{m} / \mathrm{s}$.

\subsection{ANN methodologies}

Equally important to an adequate parameterization of the used forecasting methodology is the suitable interpretation of the used data. Time series' data can be interpreted in many different ways, and data sequences can be looked at from different perspectives. For this reason various forecasting methodologies based on ANN have been developed. After exhaustive preliminary tests to choose the most suitable forecast input data, three

Tiago Pinto, Luis Marques, Tiago M. Sousa, Isabel Praça, Zita Vale and Samuel L. Abreu Data-Mining-based filtering to support

Solar Forecasting Methodologies

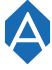

ADCAIJ: Advances in Distributed Computing and Articial Intelligence Journal Regular Issue, Vol. 6 N. 3 (2017), 85-102 eISSN: 2255-2863 - http://adcaij.usal.es (c) Ediciones Universidad de Salamanca - cC BY 
promising solutions have been found. The authors have decided to implement these three solutions with the goal of studying and concluding which would achieve the best performance. These three solutions, or methodologies, use as input data:

- M1 - last 4 periods, i.e. use the 4 hours before the time that is intended to forecast;

- M2 - last 24 periods, i.e. use the 24 hours preceding the time of day that is intended to forecast;

- M3 - last 7 days, i.e., using data exclusively from the same hour that is intended to forecast, but corresponding the 7 previous days to the day intended to forecast.

Moreover, another issue concerning data types (fields) that influence the solar intensity has emerged. As already mentioned, the fields that are part of the historical records of solar data are: I_Glob_H (Global Horizontal Irradiance), I_Beam_N (Direct Normal Irradiance), I_Diff_H (Diffuse Horizontal Irradiance), I_Extr_H (Extraterrestrial Horizontal Irradiance), Temp (Temperature), Rel_Humidity (Humidity), Wind_Speed (Wind speed).

In order to implement, test and take conclusions, four different sets of fields have been used in the forecasting process. This way it is possible to realize which fields provide added value for the forecasting process. The four sets are:

- SM1 - only each of the four solar intensity fields independently (I_Global_H, I_Beam_N, I_Diff_H or I_Extr_H);

- SM2 - the four solar fields simultaneously (I_Global_H, I_Beam_N, I_Diff_H and I_Extr_H);

- SM3 - all fields (I_Global_H, I_Beam_N, I_Diff_H, I_Extr_H, Temp, Rel_Humidity and Wind_Speed);

- SM4 - The global irradiance field (I_Global_H) used with the three complementary fields (Temp, Rel_ Humidity and Wind_Speed).

Thus, each of the three methodologies (last 4 periods, last 24 periods, and last 7 days), is subjected to four sub-methodologies (SM) based on the 4 datasets that were described before.

The sensitivity analysis consisted in a huge amount of tests, with the purpose of reaching the most advantageous combination of parameters. The parameters that have presented the higher influence on the results are: the number of nodes in the ANN's hidden layer, and the training limit, i.e. the amount of training data. Figure 1 presents the results of the variation of the number of intermediate layer nodes, when using each of the four fields of solar irradiance independently for the forecast, namely: I_Global_H, I_Beam_N, I_Diff_H and I_Extr_H. Figure 2 presents the SMAPE error variation for different amounts of training data.

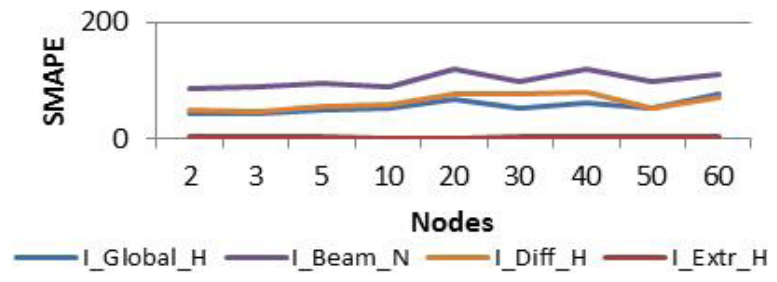

Figure 1. Forecasting error for different numbers of intermediate nodes, for each solar field

From Figure 1 and Figure 2 it is visible that the best combination would be to use 3 intermediate nodes with a training limit of 120 . Figure 1 shows that the increase of the hidden layer nodes instigates an increase of the forecasting error values in three from the four solar intensity data types. 3 nodes is the number that presents the best overall results for the four fields. Regarding the training limit, one can see from Figure 2 that the SMAPE values stabilize after the value of 120 . This means that it is irrelevant to include a larger amount of training data, as the increase in training execution time does not bring any added value for the quality of the forecasts.

Tiago Pinto, Luis Marques, Tiago M. Sousa, Isabel Praça, Zita Vale and Samuel L. Abreu Data-Mining-based filtering to support

Solar Forecasting Methodologies
ADCAIJ: Advances in Distributed Computing and Articial Intelligence Journal Regular Issue, Vol. 6 N. 3 (2017), 85-102 elSSN: 2255-2863 - http://adcaij.usal.es (c) Ediciones Universidad de Salamanca - CC BY 


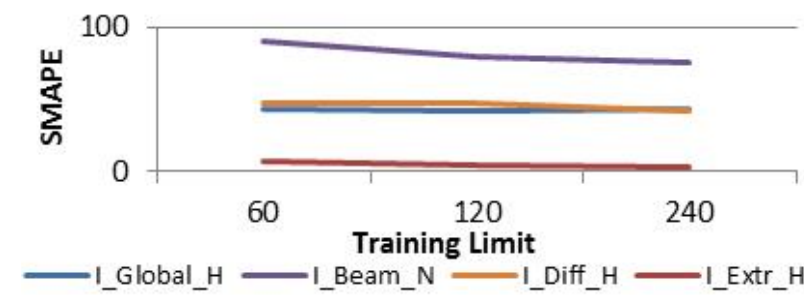

Figure 2. Forecasting error for different amounts of training data, for each solar field

Thus 3 intermediate layer nodes, and a training limit of 120 are the values that are used for all ANN based methodologies. Table 1 shows the SMAPE error values of the first methodology (M1).

Table 1. SMAPE error values obtained in the forecasts using M1 (with the last 4 periods)

\begin{tabular}{lllll}
\hline & I_Glob_H & I_Beam_N & I_Diff_H & I_Extr_H \\
\hline M1 - SM1 & 39,81 & 81,49 & 38,24 & 1,16 \\
M1 - SM2 & 37,22 & 76,19 & 47,68 & 7 \\
M1 - SM3 & 43,67 & 81,82 & 34,6 & 8,16 \\
M1 - SM4 & 40,14 & - & - & - \\
\hline
\end{tabular}

In SM4 only the I_Glob_H field is forecasted, therefore, Table 1 does not show the error value concerning the other solar intensity fields. For M1, one can see that the best results in forecasting the I_Glob_H field are achieved when using the four solar intensity fields at the same time, for the forecasting process (SM2). Table 2 shows the SMAPE forecasting results, referring to the second methodology (M2).

Table 2. SMAPE error values of the M2 methodology

\begin{tabular}{lllll}
\hline & I_Glob_H & I_Beam_N & I_Diff_H & I_Extr_H \\
\hline M2 - SM1 & 34,02 & 74,81 & 46,14 & 2,69 \\
M2 - SM2 & 54,57 & 100,6 & 47,43 & 7,86 \\
M2 - SM3 & 47,43 & 92,87 & 92,81 & 9,92 \\
M2 - SM4 & 46,27 & - & - & - \\
\hline
\end{tabular}

From Table 2 it is visible that, concerning the forecast errors' analysis using the last 24 periods, using only one solar field (SM1) leads to obtaining better predictions. Table 3 presents the results of M3.

Table 3. SMAPE error of the forecasts using the last 7 days - M3

\begin{tabular}{lllll}
\hline & I_Glob_H & I_Beam_N & I_Diff_H & I_Extr_H \\
\hline M3 - SM1 & 44,4 & 83,08 & 61,4 & 0,52 \\
M3 - SM2 & 48,96 & 74,28 & 49,72 & 8,67 \\
M3 - SM3 & 53,19 & 109,87 & 49,03 & 9,91 \\
M3 - SM4 & 39,86 & - & - & - \\
\hline
\end{tabular}

Tiago Pinto, Luis Marques, Tiago M. Sousa, Isabel Praça, Zita Vale and Samuel L. Abreu Data-Mining-based filtering to support

Solar Forecasting Methodologies
ADCAIJ: Advances in Distributed Computing and Articial Intelligence Journal Regular Issue, Vol. 6 N. 3 (2017), 85-102 elSSN: 2255-2863 - http://adcaij.usal.es (c) Ediciones Universidad de Salamanca - CC BY 
From Table 3, concerning the forecasts errors' analysis with the last 7 days, we concluded that using one solar field (SM1) leads to obtaining better forecasts, precisely because it gets the best global solar intensity forecast.

Finally, to conclude the ANN tests analysis, in the first methodology, using the last 4 periods it was concluded that using the 4 solar fields obtain better forecasts, with and error of 37,22 for I_Glob_H parameter. In the second methodology, using the last 24 periods best forecasts are obtained using one solar field, with an error of 34,02 for the parameter I_Glob_H. Finally, the third methodology using the same period of last week, obtains better predictions using one solar field, with an error of 44,4 for the parameter I_Glob_H. The methodology which achieved better forecast, as can be seen by calculating the error, was the second, using the last 24 periods, and the first sub-methodology, using only the I_Glob_H field as training data, while ignoring the other (M2 SM1), with a SMAPE value of 34,02 .

\subsection{SVM methodologies}

Similarly to the ANN based methodologies, more than one approach has been considered, regarding the input data to train the SVM. Two solutions have been implemented, which use as input:

- SVM_M1 - the same period in the last days, i.e., using data from the same hour that is forecasted, but in the last days preceding the day to forecast;

- SVM_M2 - last hours, i.e. use the latest hours before the hour of the day that is being forecasted.

Considering the conclusion taken from the performance of the ANN based approach that the use of the I_Glob_H field by itself leads to better forecasting results, and given the intrinsic nature of SVM, which assumes a single data series prediction; only the historical data of the I_Glob_H field is used by the SVM based approaches.

Sensitivity tests have been performed in order to determine the best parameterizations for the SVM approach. The most influential parameters on the results are: the kernel function, the angle of the kernel function $-\sigma$, and the amount of training data - training limit. Regarding the kernel functions, as mentioned before, the most suitable kernel functions for time series prediction are the RBF and eRBF kernels; therefore, these two kernels have been used.

Figure 3 and Figure 4 present the evolution of the MAE and SMAPE error values for different training limits and $\sigma$ respectively, when using the SVM approach with the RBF kernel.

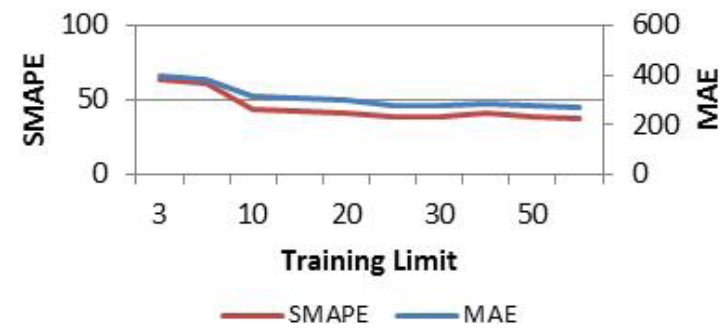

Figure 3. Forecasting error for different training limits, when using the SVM approach with the RBF kernel

Tiago Pinto, Luis Marques, Tiago M. Sousa, Isabel Praça, Zita Vale and Samuel L. Abreu Data-Mining-based filtering to support

Solar Forecasting Methodologies
ADCAIJ: Advances in Distributed Computing and Articial Intelligence Journal Regular Issue, Vol. 6 N. 3 (2017), 85-102 elSSN: 2255-2863 - http://adcaij.usal.es (c) Ediciones Universidad de Salamanca - CC BY 


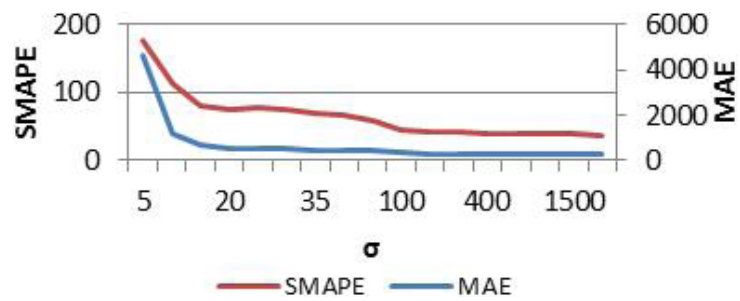

Figure 4. Forecasting error for different kernel function angles, when using the SVM approach with the RBF kernel

From Figure 3 and Figure 4 it can concluded that the use of the SVM methodology with the RBF kernel achieves the best results with a training limit of 25 and $\sigma$ equal to 1000. Figure 5 and Figure 6 present similar sensitivity analysis results for the SVM approach using the eRBF kernel.

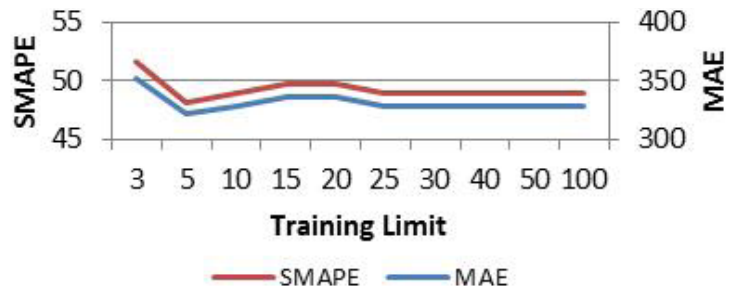

Figure 5. Forecasting error for different training limits, when using the SVM approach with the eRBF kernel

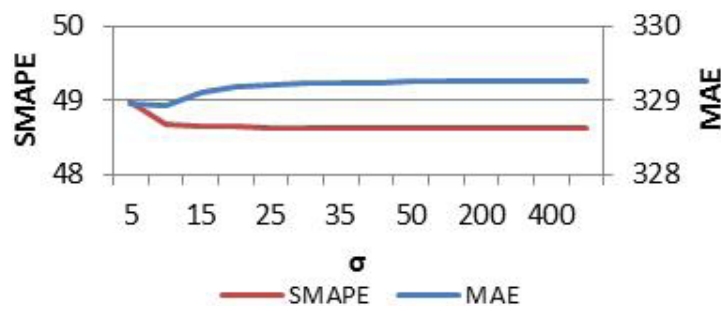

Figure 6. Forecasting error for different kernel function angles, when using the SVM approach with the eRBF kernel

From Figure 5 and Figure 6 can be concluded that the SVM methodology using the eRBF kernel reaches its optimal performance with the training limit value of 25 and $\sigma$ of 10. Table 4 presents the Standard Deviation (SD), MAE and SMAPE error values of the SVM methodology for the I_GLOB_H solar irradiance field.

Table 4. SMAPE error of the forecasts using the last 7 days $-M 3$

\begin{tabular}{lllll}
\hline Methodology & Kernel & SD & MAE & SMAPE \\
\hline \multirow{2}{*}{ SVM_M1 } & RBF & 229,5 & 270,77 & 38,37 \\
& eRBF & 301,56 & 328,97 & 48,99 \\
\multirow{2}{*}{ SVM_M2 } & RBF & 317,12 & 179,7 & 23,36 \\
& eRBF & 287,4 & 151,62 & 21,48 \\
\hline
\end{tabular}

From Table 4 it is visible that the second methodology (SVM_M2) achieves better forecasting results than SVM _M1, for both kernel functions. Additionally, despite the use of the RBF kernel being able to provide better results with the SVM_M1 methodology, the eRBF kernel was able to achieve better results with the SVM_M2 
methodology, and also the global best ones of the SVM based methodologies. Therefore, the conclusion is that using SVM_M2 with the eRBF kernel is the solution capable of reaching the best solar irradiance forecasts.

Finally, comparing the SVM approach with the ANN methodologies (which best result has been achieved by the M2 - SM1 methodology, with a SMAPE value of 34,02), one can conclude that the SVM_M2 methodology with the eRBF kernel is the best overall approach, with a SMAPE of 21,48.

\subsection{Hybrid methodologies}

The huge differences of forecasting quality that have been observed in the previous tests when using different methodologies, support the fact that the way data is looked at when analysing the historic, is essential for the forecasting process. For this reason, a novel approach is proposed, which aims at filtering and arranging the data automatically, selecting only the data that is most relevant for each case, so that the disparity of historic values can be reduced, and only the most influential and constant data is used for the training process.

The data filtering process is conducted by applying a clustering algorithm, k-means, which groups the data into different clusters according to the values similarity. Several different approaches for splitting the historical data have been experimented, namely, by year, by season, by month, and by periods of the day.

In order to evaluate the best grouping of the clustering mechanism CDI (Clustering Dispersion Indicator) is used. CDI calculates the disparity of the values that are grouped in the same cluster, therefore, the smaller the value is, the most constant the values of each cluster are, i.e. the used data set becomes less volatile. Figure 7 presents the CDI values that have been achieved, for a different number of clusters, for each different grouping approach.

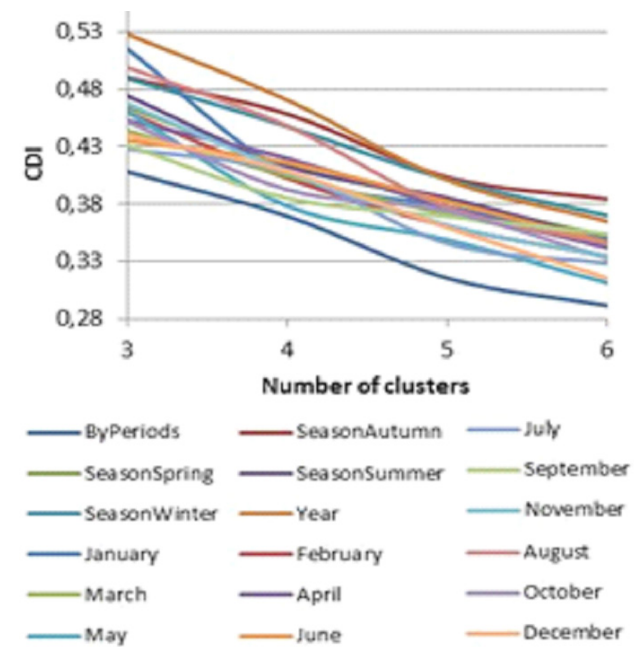

Figure 7. CDI value for each data grouping approach according to number of clusters

From Figure 7 it becomes conclusive that the division by periods of the day is the best one, as it is clearly below all other approaches for all numbers of clusters. The splitting by periods of the day means that data is divided by hours of the day. In the current case, of solar intensity forecasting, the data profile for each day is similar throughout the time: null values during night-time; an increasing tendency during the morning; the peak values are found during the midday hours; and finally a decreasing tendency until nightfall. This grouping process assures that, for instance the night-time null values are all placed in one group, the peak hours of solar irradiance in another, and so on. This way, when forecasting a solar irradiance value for a certain hour of the day, only the data concerning the most similar hours are used, refraining from using irrelevant (or even misleading) data from the training process.

The number of clusters that have been used for these tests is 6 . A careful balance between the number of clusters and the possible gain by using them must be taken into account. Note that using a high number of clusters means keeping a smaller amount of historic data for the training process. On the other hand, the higher the number of clusters is, the more refined the grouping is. For this reason, one must realize at which point the use 
of additional clusters stops being advantageous. This is usually verified by determining the point in which the CDI curve starts stabilizing and decreasing less drastically. For the current case the ideal number is 6 clusters, as can be seen by the ByPeriods line of the graph of Figure 7. Figure 8 presents the clustering results of the ByPeriods approach using five clusters, for twelve exemplification days (1st day of each month of the year 1999).

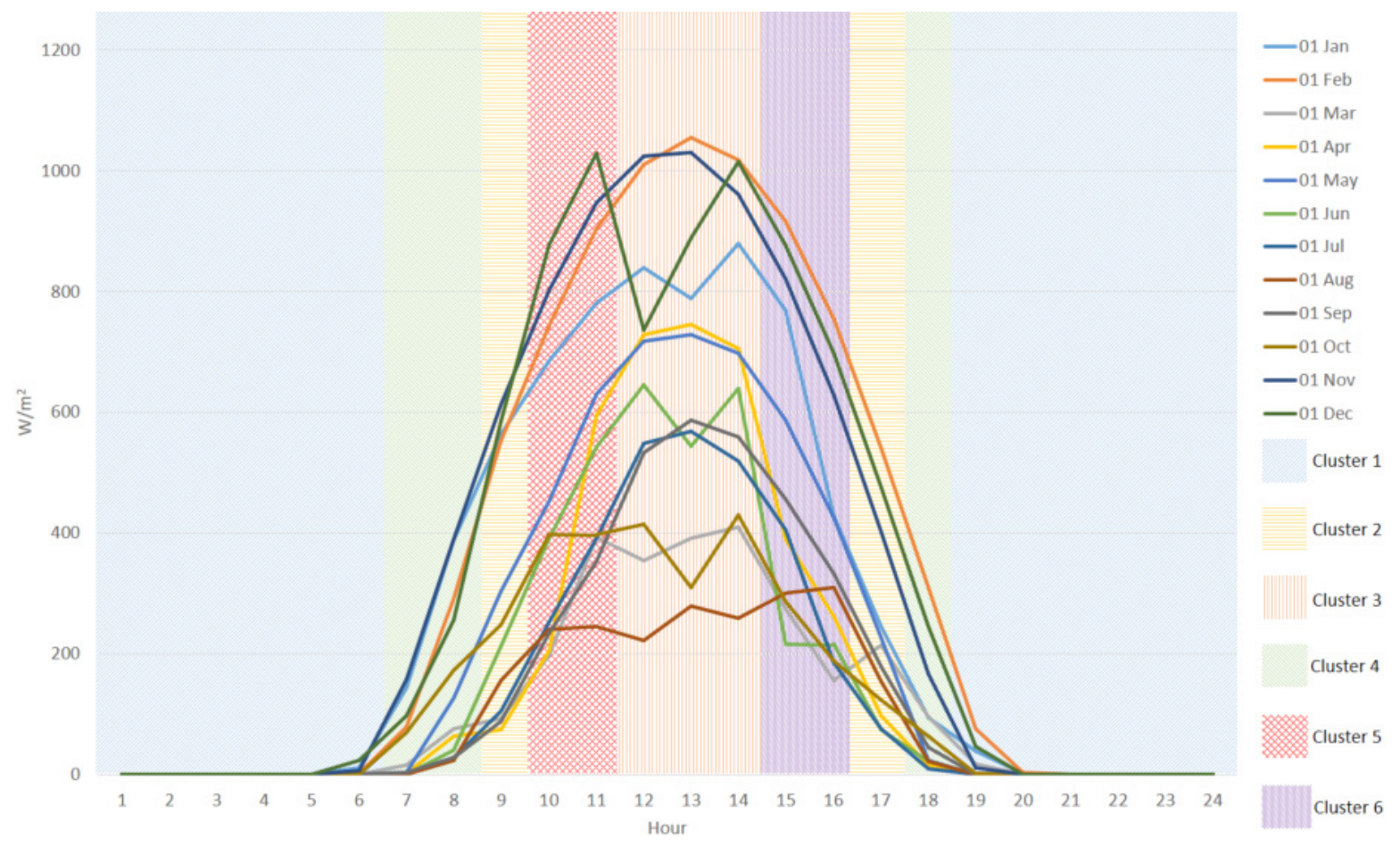

Figure 8. Clustering results of the ByPeriods approach, for six clusters

From Figure 8 it is visible that hours with similar solar intensity values are grouped in the same cluster. This is true even for days with very different values of solar intensity, such as days during the winter and during the summer. Since the solar tendency is similar during all year, all days are successfully classified in clusters that join nightly hours (null values), peak intensity hours, and hours with intermediate solar intensity. Using the separation of hours provided by the clustering process, the forecasting process is adapted, so that only the hours from previous days that are classified in the same cluster are considered as training data by the ANN and the SVM approaches.

Table 5 presents the comparison between the SMAPE error values of the best ANN and SVM approaches, with and without the use of the proposed filtering process.

Table 5. SMAPE error of the forecasts of the best ANN and SVM methodologies with and without the use of the proposed clustering based filtering process

\begin{tabular}{llll}
\hline Methodology & Kernel & Without Clustering & With Clustering \\
\hline ANN M2_SM1 & - & 34,02 & 29,71 \\
\multirow{2}{*}{ SVM_M2 } & RBF & 23,36 & 21,84 \\
& eRBF & 21,48 & 19,23 \\
\hline
\end{tabular}

From Table 5 it is visible that the use of the data filtering process implicates an increase in the forecasting quality of the proposed ANN and SVM approaches. The decrease of the error values is originated by an adequate selection of the data that is used for the training process.

Tiago Pinto, Luis Marques, Tiago M. Sousa, Isabel Praça, Zita Vale and Samuel L. Abreu Data-Mining-based filtering to support

Solar Forecasting Methodologies

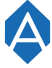

ADCAIJ: Advances in Distributed Computing and Articial Intelligence Journal Regular Issue, Vol. 6 N. 3 (2017), 85-102 eISSN: 2255-2863 - http://adcaij.usal.es (C) Ediciones Universidad de Salamanca - CC BY 


\subsection{Execution time}

The computational effort has been measured for both ANN and SVM methodologies. The parameter that, obviously, presents the higher influence over the execution time of both approaches is the training limit.

All tests have been executed on a machine with the following characteristics: Intel ${ }^{\circledR}$ Xenon $\AA$ CPU X5450 $3,00 \mathrm{Ghz}$ (2 processors), 4,00GB of RAM memory and a 32bits operating system.

Figure 9 presents the evolution of the average execution time after 1000 runs for the ANN M2-SM1 methodology.

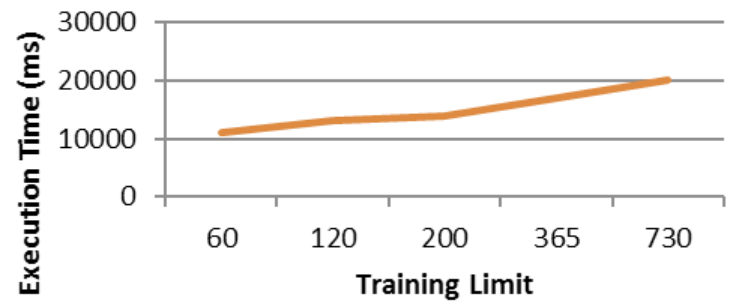

Figure 9. Average execution time of the ANN M2-SM1 methodology

From Figure 9 is it visible that the execution time increases with the increase of the training limit. As presented in section IV A, the ANN M2-SM1 methodology has been executed with a training limit of 120, which means an execution time of approximately $13500 \mathrm{~ms}$. The use of an higher training limit can produce a computational cost of $20000 \mathrm{~ms}$, while using a very low training limit takes nearly $10000 \mathrm{~ms}$ to execute.

Figure 10 presents the average execution time after 1000 runs, for the SVM based methodologies.

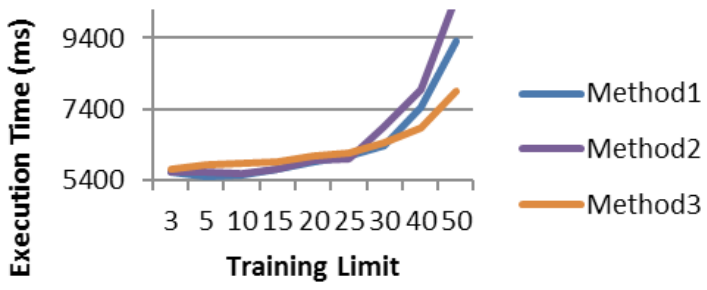

Figure 10. Average execution time for methodologies based on SVM(Metodologyl that uses the same period of the last days, Metodology2 that uses the latest hours of day and Metodology3 where Clustering is applied), depending on the Training Limit

From Figure 10 it is visible that the SVM based approaches using the training limit of 25 (the optimal value for both kernels as presented in section IV B) require nearly $6000 \mathrm{~ms}$ to run. This value is less than half of the time of the ANN based approaches. Even with a very high training limit, the execution time of the SVM approaches is always lower than the faster ANN approaches.

\subsection{Summary}

From all the presented tests one can conclude that, for the approached problem of solar intensity forecasting, the use of additional data fields other than the I_Glob_H historic values, brings no added value to the forecasting process. In fact, the forecasting error increases when using additional information.

The ANN based methodology that achieved the best results uses the last 24 periods, and the first sub-methodology, using only the I_Glob_H field as training data, while ignoring the other (M2 - SM1), with a SMAPE value of 34,02 .

Tiago Pinto, Luis Marques, Tiago M. Sousa, Isabel Praça, Zita Vale and Samuel L. Abreu Data-Mining-based filtering to support

Solar Forecasting Methodologies
ADCAIJ: Advances in Distributed Computing and Articial Intelligence Journal Regular Issue, Vol. 6 N. 3 (2017), 85-102 elSSN: 2255-2863 - http://adcaij.usal.es (c) Ediciones Universidad de Salamanca - cC BY 
Regarding the SVM based methodologies, the eRBF kernel has shown to be the most suitable for this case. While the second methodology, using only the last hours before the hour of the day that is being forecasted, achieved the best results. The SVM approaches have achieved better forecast results than the ANN.

The application of the proposed clustering based filtering process brought a visible improvement for both ANN and SVM based approaches, by selecting the data of only the most similar periods of the day to the hour of each forecast. The proposed methodology was able to decrease the SMAPE error value to 19,23: the best value achieved in all of the considered tests. This value has been achieved by applying the proposed data filtering method to the SVM_M2 methodology.

Finally, regarding the execution time of the considered approaches, the SVM based methodologies present an execution time of about half the value of the ANNs. The application of the clustering mechanism does not show any visible increase in the execution time over a large number of executions, since it is only executed once.

\section{Conclusions}

This paper presented a new approach to filter the most relevant data to be used in the training process of ANN and SVM based solar intensity forecasting methodologies.

The proposed methodology uses a clustering algorithm to group the historic data into different clusters, according to its similarity. This way the disparity of training values is reduced, and the forecast process is facilitated.

Results show that the application of the proposed method enables ANN and SVM based approaches to improve the forecasting results quality, with no significant increase of the methodologies' execution time.

Comparing the performance of the SVM based approaches with the ANN based methodologies, the higher quality of the SVM forecasts is evident, enhanced by the achievement of results in roughly half the time of the ANNs. The eRBF is the kernel function that has shown the best quality of results.

Regarding the use of complementary data fields, such as solar components other than the global irradiance, or other environmental information such as temperature, humidity and wind speed, their inclusion in the training process showed to be unnecessary, leading to a degradation of forecasts quality.

As future work, the use of other forecasting methodologies, such as fuzzy inference systems can be mentioned. Additionally, the refinement of the ANN and SVM methodologies may lead to an improvement of the results.

\section{Acknowledgements}

This work has been developed under the European Union's Horizon 2020 research and innovation programme, Marie Sklodowska-Curie grant agreement No 703689 (project ADAPT); EUREKA - ITEA2 Project FUSE-IT (ITEA-13023) and Project GREEDI (ANI|P2020 17822).

\section{References}

Alessandrini, S. Delle Monache, L. Sperati, S. Cervone, G. An analog ensemble for short-term probabilistic solar power forecast, Applied Energy, Volume 157, 1 November 2015, pp. 95-110.

Badescu, V. «Modeling solar radiation at the earth surface», Springer (2008).

Barzin, R., Chen, J.J. Young, B.R., Farid, M.M., «Application of weather forecast in conjunction with pricebased method for PCM solar passive buildings - An experimental study», Applied Energy, Volume 163, 2016, pp. 9-18.

Tiago Pinto, Luis Marques, Tiago M. Sousa, Isabel Praça, Zita Vale and Samuel L. Abreu Data-Mining-based filtering to support

Solar Forecasting Methodologies
ADCAIJ: Advances in Distributed Computing and Articial Intelligence Journal Regular Issue, Vol. 6 N. 3 (2017), 85-102 elSSN: 2255-2863 - http://adcaij.usal.es (c) Ediciones Universidad de Salamanca - cC BY 
Bian, W.; Chen, X., «Neural Network for Nonsmooth, Nonconvex Constrained Minimization Via Smooth Approximation,» Neural Networks and Learning Systems, IEEE Transactions on , vol. 25, no. 3, pp. 545-556, March 2014.

Boser, B.E., Guyon, I.M., Vapnik, V.N., «A Training algorithm for optimal margin classifiers», COLT conference, 1992.

Chicco G., Ilie S., «Support Vector Clustering of Electrical Load Pattern Data». IEEE Transactions on Power Systems, vol. 24, no. 3, pp. 1619-1628, August 2009.

Diagne, M., David, M., Lauret, P., Boland, J., Schmutz, N., «Review of solar irradiance forecasting methods and a proposition for small-scale insular grids», Renewable and Sustainable Energy Reviews, Elsevier, vol 27, pp. 65-76, 2013.

Dianhui W.; Tapan, S., «A Robust Elicitation Algorithm for Discovering DNA Motifs Using Fuzzy Self-Organizing Maps,» Neural Networks and Learning Systems, IEEE Transactions on , vol. 24, no. 10, pp. 16771688 , Oct. 2013

European Commissison, »The 2020 climate and energy package ». Available at http://ec.europa.eu/clima/policies/package/index en.htm, last accessed August 2017.

Fisher, R., «The use of multiple measurements in taxonomic problems. Annals of Eugenics», 7, 111-132, 1936.

Gupta, R., Gupta, G.; Kastwar, D.; Hussain, A.; Ranjan, H.; »Modeling and design of MPPT controler for a PV model using PSCAD/EMTDC». Innovative Smart Grid Technologies Conference Europe (ISGT-Europe), 2010 IEEE PES, 11-13 October 2010.

Han, J. and Kamber, M. «Data mining: concepts and techniques». The Morgan Kaufmann series in data management systems, San Francisco, 2006.

Hao Q; Srinivasan, D.; Khosravi, A., «Short-Term Load and Wind Power Forecasting Using Neural Network-Based Prediction Intervals,» Neural Networks and Learning Systems, IEEE Transactions on, vol. 25, no. 2, pp. 303-315, Feb. 2014

Hocaoglu, F.O., Serttas, F., A novel hybrid (Mycielski-Markov) model for hourly solar radiation forecasting, Renewable Energy, Volume 108, August 2017, pp. 635-643

Huynh T. Q. and Reggia J. A., «Symbolic Representation of Recurrent Neural Network Dynamics,» IEEE Trans. Neural Networks Learn. Syst., vol. 23, no. 10, pp. 1649-1658, Oct. 2012.

Hyndman, J., Koehler, B., «Another look at measures of forecast accuracy. International journal of forecasting», $22(4), 679-688,2006$.

Inman, Rich H., Pedro, Hugo T. C., Coimbra, Carlos R. M., «Solar forecasting methods for renewable energy integration», Progress in Energy and Combustion Science, Elsevier, vol. 39, pp. 535-576, 2013.

Ioakimidis, C., et al., «Solar Production Forecasting Based on Irradiance Forecasting Using Artificial Neural Networks», 39th Annual Conference of the IEEE Industrial Electronics Society (IECON 2013), pp. 8121 8126, Nov 2013.

Jain A. K.. «Data Clustering: 50 years beyond K-Means». Pattern Recognition Letters, Elsevier, Vol. 31, Issue 8, pp. 651-666, June 2010.

Jain, A. K., Murty M. N. and Flynn, P. J. (1999) Data Clustering: A Review. In: ACM Computing Surveys, 31 (3). pp. 264-323.

Keles, D., Scelle, J., Paraschiv, F., Fichtner, W., Extended forecast methods for day-ahead electricity spot prices applying artificial neural networks, Applied Energy, Volume 162, 15 January 2016, pp. 218-230

Kopp, G. and Lean, J. L., A new, lower value of total solar irradiance: Evidence and climate significance, Geophysical Research Letters, VOL. 38, L01706, 2011

Liu, H., Tian, H., Liang, X., Li, Y. «Wind speed forecasting approach using secondary decomposition algorithm and Elman neural networks», Applied Energy, Volume 157, 1 November 2015, pp. 183-194

Liu, N., Tang, Q., Zhang, J., Fan, W., Liu, J. «A hybrid forecasting model with parameter optimization for short-term load forecasting of micro-grids», Applied Energy, Volume 129, 15 September 2014, pp. 336-345

Martin, L. Zarzalejo, L. Polo, J. Navarro, A. Marchante, R. Cony, M. «Prediction of global solar irradiance based on time series analysis: application to solar thermal power plants energy production planning», Solar Energy, 84 (2010), pp. 1772-1781.

Tiago Pinto, Luis Marques, Tiago M. Sousa, Isabel Praça, Zita Vale and Samuel L. Abreu

Data-Mining-based filtering to support

Solar Forecasting Methodologies
ADCAIJ: Advances in Distributed Computing and Articial Intelligence Journal Regular Issue, Vol. 6 N. 3 (2017), 85-102 elSSN: 2255-2863 - http://adcaij.usal.es (c) Ediciones Universidad de Salamanca - CC BY 
Mohanty, S., Patra, P. K., Sahoo, S. S., Mohanty, A. «Forecasting of solar energy with application for a growing economy like India: Survey and implication», Renewable and Sustainable Energy Reviews, Volume 78, October 2017, pp. 539-553

Nikulin, M.S., «Loss function», in Hazewinkel, Michiel, Encyclopedia of Mathematics, Springer, 2001.

Paolik C, Voyant C, Muselli M, Nivet M. Solar radiation forecasting using ad-hoc time series preprocessing and neural networks. In: Proceeding of the 5th international conference on emerging intelligent computing technology and applications, Ulsan, South Korea; 2009, pp. 898-907.

Pedro H. T. C. and Coimbra, C. F. M. «Assessment of forecasting techniques for solar power production with no exogenous inputs», Solar Energy, Elsevier, vol. 86, pp. 2017-2028, 2012.

Pelland, S., Remund, J., Kleissl, J., Oozeki, T., De Brabandere, K. «Photovoltaic and Solar Forecasting: State of the Art», International Energy Agency Photovoltaic Power Systems Programme, 2013. ISBN: 978-3-906042-13-8.

Persson, C. Bacher, P. Shiga,T., Madsen, H. «Multi-site solar power forecasting using gradient boosted regression trees», Solar Energy, Volume 150, 1 July 2017, pp. 423-436

Pinto, T. , Ramos, S., Sousa, T. M., Vale, Z. «Short-term wind speed forecasting using Support Vector Machines», 2014 IEEE Symposium on Computational Intelligence in Dynamic and Uncertain Environments (CIDUE), 2014.

Pinto, T. et. al, «Solar Intensity Characterization using Data-Mining to support Solar Forecasting», 12th International Conference in Distributed Computing and Artificial Intelligence, Advances in Intelligent Systems and Computing, vol. 373, pp. 193-201, Springer International Publishing, 2015

Pinto, T., Sousa, T.M., Praça, I., Vale, Z., Morais,H. Support Vector Machines for decision support in electricity markets' strategic bidding, Neurocomputing, Volume 172, 8 January 2016, pp. 438-445

Schaefer A. M., Udluft S., and Zimmermann H.-G., «A Recurrent Control Neural Network for Data Efficient Reinforcement Learning,» in 2007 IEEE International Symposium on Approximate Dynamic Programming and Reinforcement Learning, 2007, pp. 151-157.

Schwaegerl, C. and Tao, L. (2013) The Microgrids Concept, in Microgrids: Architectures and Control (ed N. Hatziargyriou), John Wiley and Sons Ltd, Chichester, United Kingdom.

Sharma N., Sharma, P., Irwin, D., and Shenoy, P. «Predicting Solar Generation from Weather Forecasts Using Machine Learning», IEEE International Conference on Smart Grid Communications (SmartGridComm), pp. 528-533, October 2011.

Singh, V.P., Vijay, V., Bhatt, M. S., Chaturvedi, D. K. «Generalized neural network methodology for short term solar power forecasting», IEEE 13th International Conference on Environment and Electrical Engineering (EEEIC), pp. 58-62, Nov. 2013.

Sioshansi, F.P., «Evolution of Global Electricity Markets - New paradigms, new challenges, new approaches», Academic Press, 2013

Smola, A.,Schölkopf, B. «A tutorial on support vector regression», Statistics and Computing, 14, 199-222, 2004.

Vapnik, V., A. Lerner, A., «Pattern recognition using generalized portrait method. Automation and Remote Control», 24, 774-780, 1963.

Voyant, C. et al., Machine learning methods for solar radiation forecasting: A review, Renewable Energy, Volume 105, May 2017, pp. 569-582

Wilamowski B. M. and Yu H., «Neural network learning without backpropagation.,» IEEE Trans. Neural Netw., vol. 21, no. 11, pp. 1793-803, Nov. 2010.

Xu, R., Chen, H. and Sun, X.«Short-term Photovoltaic Power Forecasting with Weighted Support Vector Machine», IEEE International Conference on Automation and Logistics (ICAl-2012), pp. 248-253, August 2012

Zeng, J., Qiao, W. «Short-term solar power prediction using a support vector machine», Renewable Energy, Elsevier, vol. 52, pp. 118-127, 2013.

Zhao, J. et al., An improved multi-step forecasting model based on WRF ensembles and creative fuzzy systems for wind speed, Applied Energy, Volume 162, 15 January 2016, pp. 808.

Tiago Pinto, Luis Marques, Tiago M. Sousa, Isabel Praça, Zita Vale and Samuel L. Abreu

Data-Mining-based filtering to support

Solar Forecasting Methodologies
ADCAIJ: Advances in Distributed Computing and Articial Intelligence Journal Regular Issue, Vol. 6 N. 3 (2017), 85-102 eISSN: 2255-2863 - http://adcaij.usal.es (c) Ediciones Universidad de Salamanca - cC BY 